\title{
MODEL OF SLOWING THE SPREAD OF GYPSY MOTH (LEPIDOPTERA: LYMANTRIIDAE) WITH A BARRIER ZONE
}

\author{
Alexei A. Sharov ${ }^{1}$ And Andrew M. Liebhold ${ }^{2}$ \\ ${ }^{1}$ Department of Entomology, Virginia Polytechnic Institute and State University, Blacksburg, Virginia 24061 USA \\ ${ }^{2}$ Northeastern Forest Experiment Station, USDA Forest Service, 180 Canfield Street, \\ Morgantown, West Virginia 26505 USA
}

\begin{abstract}
When attempts to eradicate an introduced pest species fail and it becomes established, barrier zones are often used to stop or to slow the spread of the population into uninfested areas. The U.S. Forest Service is currently conducting a Slow-the-Spread (STS) pilot project to evaluate the feasibility of slowing the spread of the gypsy moth (Lymantria dispar L.) in several areas along the population front. To predict the effect of barrier zones on the rate of gypsy moth spread we developed a model that assumes establishment of isolated colonies beyond the expanding population front. These colonies grow, coalesce, and thereby contribute to the movement of the population front. The model estimates the rate of spread from two functions: (1) colonization rate as a function of the distance from the population front and (2) population numbers in a colony as a function of colony age. Eradication of isolated colonies in a barrier zone was simulated by truncating the colonization rate function beyond a specific distance from the population front. The model predicts that eradication of isolated colonies in the STS project will result in a 54\% reduction of spread rate. The actual rate of spread in the Appalachian Mountains has declined by $59 \%$ since 1990 when eradication of isolated colonies started. Thus, model predictions were close to the observed reduction in the rate of population spread.
\end{abstract}

Key words: barrier zone; biological invasion; gypsy moth; Lymantria dispar; model; population spread.

\section{INTRODUCTION}

Biological invasions of pest species are among the most significant threats to the stability of natural and agricultural ecosystems (Liebhold et al. 1995). Quarantine and eradication are the primary methods for excluding exotic pests but when they fail and a species becomes established, it is sometimes possible to reduce adverse impacts by stopping or slowing its spread. The simplest containment strategy is the use of domestic quarantine regulations to reduce the probability of transportation to new areas. The effectiveness of these quarantines can be increased by barrier zones. A barrier zone can be defined as an area at the front of the population distribution where eradication (or suppression) activity is performed in order to prevent or to slow the spread of the population.

The barrier zone concept of slowing or stopping range expansion has been implemented several times against a variety of pest species (Dahlsten and Garcia 1989). For example, the spread of the screwworm fly (Cochliomyia hominivorax Coquerel) population has been limited by the release of sterile males in a barrier zone area (Scruggs 1975). A barrier zone along the Hudson River was used to stop the spread of the gypsy moth from 1923 to 1941 (McManus and McIntyre 1981). Although this barrier zone eventually became

Manuscript received 20 November 1996; revised 19 December 1997; accepted 20 January 1998. infested, it apparently contributed to the reduction of the rate of population spread during that period (Liebhold et al. 1992).

A barrier zone can be either stationary or moving. If the barrier zone moves in the direction of population expansion then it will be slowing the rate of spread. Slowing population spread may be substantially less expensive than stopping the spread. The benefits from slowing the spread of a pest species result from the delay in colonization of currently uninfested areas (Leuschner et al. 1996). The present value of expected pest impacts decreases when these impacts are postponed.

Our objective was to develop a model that would be suitable for predicting the effect of a barrier zone on the rate of expansion of gypsy moth (Lymantria dispar (L.)) populations in North America. The gypsy moth is a good species to use as a case study because: (1) considerable resources are currently being expended on slowing its spread through North America and (2) historical data that describe the spread of the gypsy moth are probably more extensive in both space and time than any other invading species. The gypsy moth was accidentally introduced to North America near Boston in 1869, and since that time it has been slowly expanding its range (Liebhold et al. 1989). Liebhold et al. (1992) estimated gypsy moth spread rates using the relationship between the date of first infestation and distance from the area infested in 1900. The rate of 
spread was relatively high $(9.45 \mathrm{~km} / \mathrm{yr})$ from 1900 to 1915, then from 1916 to 1965 it was low $(2.82 \mathrm{~km} / \mathrm{yr})$, and from 1966 to 1990 it increased to $20.78 \mathrm{~km} / \mathrm{yr}$ in counties where the mean minimum January temperature was $>-13.8^{\circ} \mathrm{C}$ (this area is located generally to the south from Lake Erie).

Currently the U.S. Forest Service is conducting the Slow-the-Spread (STS) pilot project to evaluate the feasibility of slowing the spread of the gypsy moth in specific portions of North Carolina, Virginia, West Virginia, and Michigan (Leonard and Sharov 1995, Leuschner et al. 1996). Also, considerable effort is expended every year on detection and eradication of gypsy moth in virtually every currently uninfested state. Success of these programs depends on our knowledge of the mechanisms of population spread. In particular, it is important to know the effect of different pest management policies on gypsy moth spread.

As part of the USDA Forest Service Appalachian Integrated Pest Management (AIPM) (1988-1992) and STS (1992-present) projects, large numbers of pheromone traps were deployed at $1-3 \mathrm{~km}$ grid spacings over a large area that encompassed the expanding gypsy moth front in the central Appalachian Mountains (Reardon 1991, Leonard and Sharov 1995). These data probably represent the most detailed characterization of the expanding front for any invading species to date.

Newly hatched gypsy moth larvae disperse by wind. Several observations at the beginning of the century showed that gypsy moth larvae can be blown several miles away from source infestations (Collins 1915). However the distribution of traveling distance was not quantified in these studies. Field experiments of Mason and McManus (1981) showed that the number of larvae captured in sticky traps decreased very rapidly with the distance from the source. More than $99 \%$ of larvae were captured within $120 \mathrm{~m}$ from the source (Mason and McManus 1981). Taylor and Reling (1986) sampled airborne gypsy moth larvae with a net fastened to an airplane. They estimated that some larvae can travel up to $19 \mathrm{~km}$ in a single dispersal episode. But the proportion of larvae that rise above forest canopy was as low as $0.3 \%$. Diffusion models based on larval dispersal can simulate the expansion of local infestations of gypsy moths (Wilder et al. 1995). However, they failed to predict the rate of spread of large-scale populations (Liebhold et al. 1992).

Mason and McManus (1981), McFadden and McManus (1991), and Liebhold et al. (1992) suggested that accidental transportation of gypsy moth egg masses and other life stages by humans is more important for the expansion of population area than is larval dispersal. The importance of egg mass transportation is supported by a documented record of 264 isolated infestations (only $18 \%$ of these infestations exceeded 2.6 $\mathrm{km}^{2}$ ), most of which were traced to the transport of life stages on furniture, firewood, equipment, etc. (McFadden and McManus 1991).
Thus, gypsy moth has both a long-distance dispersal (accidental transportation) that leads to establishment of new colonies and a short-distance dispersal (windborne movement of first instars) by which colonies increase in area. A combination of different dispersal scales is called "stratified diffusion" or "stratified dispersal" (Hengeveld 1989).

Simulation models of pest spread can be used to test the effectiveness of various barrier zone strategies. Traditional models of population spread are based on reaction-diffusion equations. Among these are the model of Skellam (1951), which combines diffusion with exponential population growth, and the contact birth models of Mollison (1977). Marsula and Wissel (1994) used a reaction-diffusion model to simulate a barrier zone that can stop the spread of the screwworm population. They estimated the minimum width of the barrier zone and the density of released sterile males that is sufficient for stopping the spread.

However, reaction-diffusion models simulate dispersal as a continuous random walk and ignore possible discrete long-distance dispersal. They cannot be used for simulating the spread of the gypsy moth, because long-distance dispersal plays a major role in the spread of this species. Integro-differential models (Van den Bosch et al. 1990, 1992) can incorporate long-distance dispersal, but their solution is known only for a homogeneous environment. Barrier zones imply spatial heterogeneity because pest management activity reduces population growth within the barrier zone, but does not affect populations outside of the barrier zone. Thus, integro-differential equations are not helpful in simulating the effect of barrier zones.

Both reaction-diffusion and integro-differential models consider dispersal at the organism level. An alternative approach would be to model the establishment of new colonies beyond the population front and to follow the growth of these colonies, which is similar to metapopulation modeling (Hanski and Gilpin 1991). Shigesada et al. (1995) used this approach to build several models of stratified dispersal in animal populations. The scattered colony model assumes independent growth of a set of colonies that expand in size and simultaneously emit long-distance dispersal propagules. A more realistic coalescing colony model assumes that new colonies become established at a specific distance from the expanding population front. When their boundary reaches the population front, then they coalesce with the primary colony and their area is added to the infested area. Although models of Shigesada et al. (1995) generate reasonable rates of population spread, they are not realistic enough to be used for simulating the effect of barrier zones. For example, according to the coalescing colony model, eradication of offspring colonies can be restricted to a very small area because new colonies are produced at a fixed distance from the population front.

In this paper we developed a new model of the ex- 


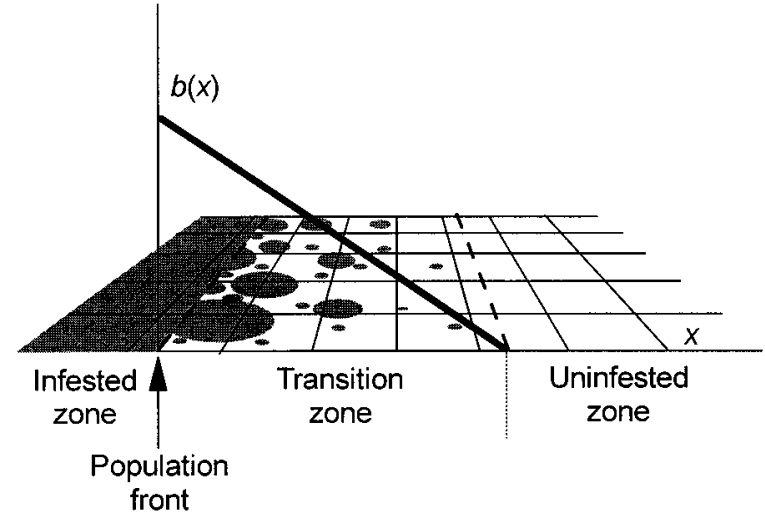

FIG. 1. Three zones at the expanding population front The probability of colony establishment, $b(x)$, decreases with increasing distance, $x$, from the front of the infested zone.

pansion of population range in which colonies can become established at varying distances from the population front. This model is biologically more realistic and provides more meaningful representation of barrier zone strategies.

\section{Model of Population Spread}

The proposed model considers the following three zones at the moving population front: the infested zone that is assumed to be a source of long-distance dispersal, the transition zone where new colonies become established, and the uninfested zone where no colonies exist (Fig. 1). New colonies become established at varying distances from the population front, which is the boundary between the infested and transition zones. We assume that the population front is always a straight line that progresses uniformly at all locations. Thus, we can build the model using just one spatial coordinate that is perpendicular to the population front.

Our objective is to calculate the rate of population spread from the following two functions: (1) the rate of colony establishment (per unit area per unit time), $b(x)$, which continuously decreases with increasing distance, $x$, from the front of the infested zone (Fig. 1) and (2) the number of individuals in a colony, $n(a)$, which continuously increases with colony age (time since colonization), $a$.

The model describes the average density per unit area of colonies, $m(a, z, t)$, of age $a$, at spatial location $z$, and at time $t$. Although natural extinction of colonies does occur, we ignored it in the model by considering only those colonies that successfully established. This makes the rate of colony establishment, $b(x)$, smaller. The density of colonies changes according to the Von Foerster's model, i.e.,

$$
\frac{\partial m}{\partial t}=-\frac{\partial m}{\partial a}
$$

(Hastings 1991, Shigesada et al. 1995). The renewal condition specifies the rate of establishment of new colonies

$$
m(0, z, t)=b[z-Z(t)]
$$

where $Z(t)$ is the location of the population front at time $t$. The average density of individuals, $N(z, t)$, at location $z$ at time $t$ is

$$
N(z, t)=\int_{0}^{\infty} m(a, z, t) n(a) d a
$$

By definition, the population front is located at the farthest point where the average density of individuals per unit area is greater than a specific density threshold, $K$, which can be interpreted as the carrying capacity:

$$
Z(t)=\max \{z \mid N(z, t) \geq K\} .
$$

We do not use parameter $K$ to limit the growth of individual colonies because we intend to simulate population dynamics only in the transition zone where the average population density is below the carrying capacity. Instead we use parameter $K$ to define the location of the population front, $Z(t)$. Then we can use Eq. 5 to predict the rate of establishment of new colonies at varying distances from the population front.

The simplifying assumption that the rate of colony establishment depends only on the distance from the population front does not imply that all long-distance dispersal originates only from the population front. Usually, isolated colonies have low densities and, numerically, are a small fraction of the population. Thus, their contribution to long-distance dispersal should be much smaller than the contribution of high-density populations that have reached the carrying capacity.

If the historical expansion of the population front is known to be $Z(t)$, for $t<t_{0}$, where $t_{0}$ is the current time, then the number of colonies, $m(a, z, t)$, of age $a$ at location $z$ is equal to the rate of colony establishment $a$ time units ago (Eqs. 1 and 2):

$$
m(a, z, t)=b[z-Z(t-a)] .
$$

Then, the average density of individuals at location $z$ is:

$$
N(z, t)=\int_{0}^{\infty} b[z-Z(t-a)] n(a) d a .
$$

The function $N(z, t)$ is continuously decreasing with increasing distance, $z \geq Z(t)$, because the rate of colony establishment, $b$, is a continuously decreasing function, and, according to Eq. $4, N(z, t)$ is finite in the transition zone. Thus, the condition in Eq. 4 can be simplified as

$$
N[Z(t), t]=K .
$$

Note that initial conditions (i.e., the function $Z(t)$, $-\infty<t \leq t_{0}$ ), cannot be selected arbitrarily, because the function $Z(t)$ should satisfy equation (7) at each point $t$. However for numerical analysis it is possible to start from an arbitrarily selected function $Z(t),-\infty$ 
$<t \leq t_{0}$, which is scaled so that the condition in Eq. 7 is satisfied for current time $\left(t=t_{0}\right)$. The average density of organisms, $N(z, t)$, is estimated using Eq. 6 and then differentiated numerically by both arguments at $t=t_{0}$, and $z=Z\left(t_{0}\right)$. Then the rate of advance of the population front is

$$
\frac{d Z}{d t}=-\frac{\partial N}{\partial t} / \frac{\partial N}{\partial z}
$$

Eq. 8 follows directly from Eq. 7 .

We consider only those functions $b(x)$ that decrease faster than exponentially with increasing distance $x$ from the population front. In this case, past values of function $Z(t)$ become "forgotten" as time progresses. Thus, the effect of the initial function $Z(t)$ on the estimated rate of spread becomes negligible after some period. Numerical simulations showed that for all specific functions $b(x)$ and $n(a)$ used in this paper, the rate of spread ultimately converged to a stationary value. Thus, it was possible to apply the traveling wave equation for estimating the rate of spread. Let us assume that the rate of spread is constant and equal to $v$. Then, $Z(t)=z_{0}+v t$. Substituting this function together with $z=Z\left(t_{0}\right)$ and $t=t_{0}$ into Eq. 6, we get

$$
N\left[Z\left(t_{0}\right), t_{0}\right]=\int_{0}^{\infty} b(a v) n(a) d a .
$$

Combining this equation with Eq. 7 we get the following traveling wave equation:

$$
\int_{0}^{\infty} b(a v) n(a) d a=K .
$$

This equation can be used for estimating the spread rate, $v$, if functions $b(x)$ and $n(a)$ are specified explicitly.

We assume that the number of individuals in a colony, $n(a)$, increases exponentially with colony age $a$ :

$$
n(a)=n_{0} \exp (r a)
$$

where $n_{0}$ is the initial number of individuals in a new colony, and $r$ is the intrinsic rate of increase. Colony establishment rate is assumed to decrease linearly with the distance from the front of the infested zone:

$$
\begin{array}{ll}
b(x)=c\left(1-\frac{x}{x_{\max }}\right) & \text { if } x<x_{\max } \\
b(x)=0 & \text { if } x>x_{\max }
\end{array}
$$

where $x_{\max }$ is the maximum distance at which colonies can become established (=width of the transition zone), and $c=b(0)$ is the maximum rate of colony establishment in the area adjacent to the infested zone (Fig. 1).

The traveling wave Eq. 10 combined with 11 and 12 yields an equation that can be used to estimate the relative rate of population spread, $V=v / x_{\max }$ :

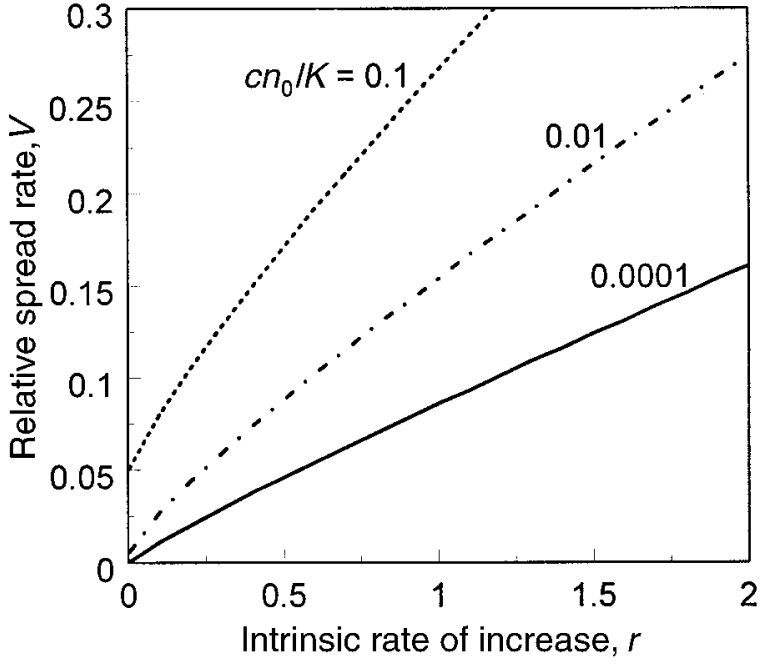

FIG. 2. Relative population spread rate $\left(V=v_{\max } / x_{\max }\right)$ predicted by the model with a linear function $b(x)$.

$$
\frac{c n_{0} V}{r^{2}}\left[\exp \left(\frac{r}{V}\right)-\frac{r}{V}-1\right]=K .
$$

Then, the rate of population spread is $v=V x_{\max }$. The rate of spread is always proportional to the width of the transition zone, $x_{\max }$. Eq. 13 cannot be solved analytically and thus we used numerical methods to estimate $V$.

The relative population spread rate, $V$, increased with increasing values of $c n_{0} / K$ (Fig. 2). The value of $c n_{0} /$ $K$ is the density of organisms in newly established colonies just beyond the front of the infested zone $\left(\mathrm{cn}_{0}\right)$ relative to the total number of organisms at the front of the infested zone $(K)$. It quantifies the intensity of the colonization process. We expect this value to be very small $(<0.1)$ in most dispersing populations, because establishment of new colonies is a rare event and the initial number of individuals $\left(n_{0}\right)$ is usually small.

The rate of population spread is positive when $r=$ 0 (Fig. 2), which seems to contradict other models of population spread (Skellam 1951, Van den Bosch et al. 1992). However, this results from a different interpretation of the parameter $r$, which in our case does not include the colonization process. If isolated colonies do not grow, the population slowly spreads forward due to the pure colonization process. For comparison with other models, it may be useful to adjust parameter $r$ using the equation: $r^{\prime}=\ln \left[\exp (r)+c n_{0} / K\right]$.

The relationship between the intrinsic rate of increase, $r$, and the rate of spread is almost linear, especially at large values of $c n_{0} / K$ (Fig. 2). The rate of spread has low sensitivity to the value of $c n_{0} / K$. For example, if $c n_{0} / K=0.1$ and $r=0.5$, then a 10 -times decrease in colonization rate, $c$, resulted only in a 2times decrease in spread rates (Fig. 2). For smaller values of $c n_{0} / K$, the sensitivity is even lower. If $c n_{0} / K$ $=0.01$ and $r=0.5$, then a 100-times decrease in col- 


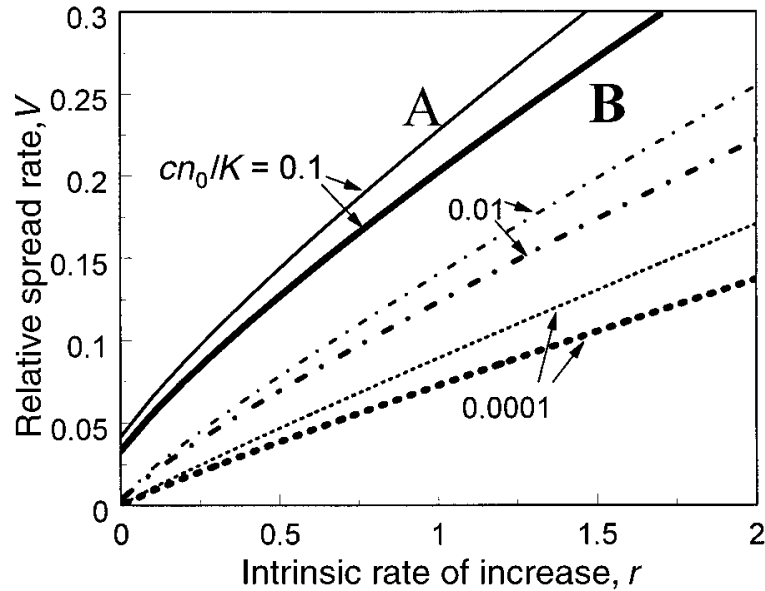

FIG. 3. Relative population spread rate $\left(V=v_{\max } / x_{\max }\right)$ predicted by the model with a $1 / 2-\operatorname{normal}(\mathrm{A})$ and a quadratic (B) function $b(x)$.

onization rate, $c$, resulted in a 2-times decrease in spread rates.

To check the sensitivity of the model to the shape of function $b(x)$ we used the following two alternative $b(x)$ functions: (1) $1 / 2$ of a normal distribution (height, $c$, and standard deviation, $x_{\max } / 3$ ) and (2) a quadratic function $b(x)=c\left(1-x / x_{\max }\right)^{2}$ for $x<x_{\max }$ and $b(x)=$ 0 for $x \geq x_{\max }$. There was no qualitative difference in the behavior of the model with various colony establishment functions (Figs. 2 and 3).

Now we introduce a barrier zone that starts at distance $x_{0}$ from the population front and extends to the end of the transition zone. Because the rate of population spread depends on the presence of a barrier zone, we will distinguish between the uncontrolled rate of spread, $v_{\max }$, and the rate of spread reduced by a barrier zone, $v_{\text {bar }}$.

Eradication of all colonies in a barrier zone is equivalent to truncating the tail of the colonization function, $b(x)$, at $x \geq x_{0}$ (Fig. 4). This truncated function can be used in the traveling wave Eq. 3 to estimate the rate of population spread, $v$, in the presence of a barrier zone. The reduction in the rate of population spread can be determined from a combination of two traveling wave equations (Eq. 10) with non-truncated and truncated linear functions $b(x)$ (Eq. 12), and exponential function $n(a)$ (Eq. 11):

$$
\begin{gathered}
\int_{0}^{v_{\max } x_{\max }} c\left(1-a v_{\max } / x_{\max }\right) n_{0} \exp (r a) d a \\
\quad=\int_{0}^{v_{\text {bar }} x_{0}} c\left(1-a v_{\text {bar }} / x_{\max }\right) n_{0} \exp (r a) d a .
\end{gathered}
$$

In the second integral, we truncated the interval of integration which is equivalent to the truncating of the $b(x)$ function. Eq. 14 yields the following equation:

$$
(1-R+Q W) \exp \left[\frac{Q(1-W)}{1-R}\right]+R-\exp (Q)=0
$$

where $R=1-v_{\mathrm{bar}} / v_{\max }$ is the relative reduction of population spread rate due to the barrier zone as a fraction of the spread rate without the barrier zone, $v_{\max } ; Q=r x_{\max } / v_{\max }$; and $W=1-x_{0} / x_{\max }$ is the relative width of a barrier zone defined by its proportion of the width of the transition zone, $x_{\max }$. Eq. 15 cannot be solved analytically, and thus, we used numerical methods to estimate $R$ as a function of $W$ for different values of parameter $Q$ (Fig. 5A).

Parameter $Q$ can be interpreted as follows. The ratio $x_{\max } / v_{\max }$ equals the amount of time that a location exists in the transition zone (without any population management). Then, $Q$ is the net rate of increase, $r$, multiplied by time in the transition zone. Thus, $\exp (Q)$ is the ratio of population numbers in a colony established at the front of the transition zone when it reaches the infested zone to the initial population numbers when it was established. The predicted efficiency of a barrier zone in slowing population spread increases with increasing values of parameter $Q$ (Fig. 5). Thus, the higher is the intrinsic rate of increase, $r$, the greater is the percentage reduction in the rate of population spread caused by a barrier zone.

Similar results were obtained using the quadratic function of colony establishment rate (Fig. 5B). The only difference is that wider barrier zones are needed to obtain the same level of reduction in the spread rate as obtained with the linear colony establishment function. We did not use the normal distribution function $b(x)$ for estimating the effect of the barrier zone, because this function has a long "tail." Thus, the width of the transition zone is infinite, and the variable $W$ cannot be estimated.

\section{Case Study: Gypsy Moth Spread}

In order to apply the described model to the spread of the gypsy moth in North America it was necessary

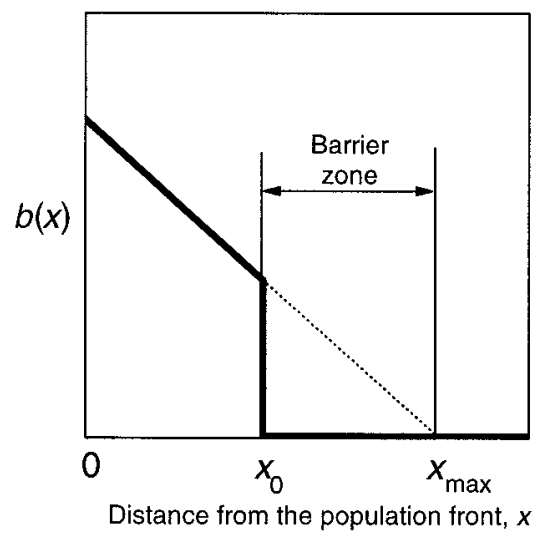

FIG. 4. The function of the probability of colony establishment, $b(x)$, becomes truncated beyond distance $x_{0}$ from the population front if a barrier zone is set in the area from $x_{0}$ to $x_{\max }$. 


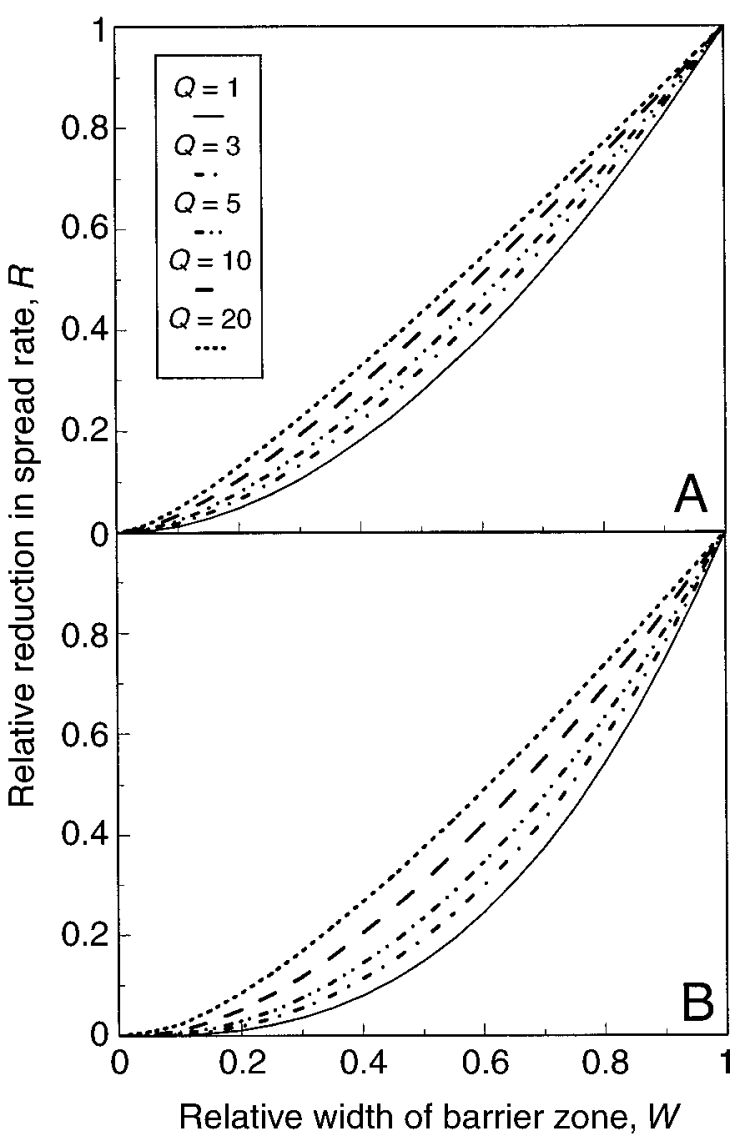

FIG. 5. Relative reduction in spread rate, $R=1-v / v_{\max }$ predicted by the model with a linear (A) and quadratic (B) function $b(x)$.

to obtain estimates of model parameters: the maximum distance from the population front where isolated colonies become established, $x_{\max }$; the rate of colony establishment at the population front, $c$; the initial number of individuals in a colony, $n_{0}$; carrying capacity, $K$; the rate of population increase, $r$; and the distance from the population front to the beginning of the barrier zone, $x_{0}$. These parameters may depend on the terrain, dominant vegetation, and other factors. Our preliminary data suggest that the spread of gypsy moth populations through the coastal plain in southeastern Virginia is different from the spread along Appalachian mountain ridges. Thus, we decided to apply the model to a specific area in the central Appalachian Mountains (Fig. 6, area between dashed lines) where gypsy moth populations have been intensively monitored since 1984 using grids of pheromone-baited traps. Most of these data were collected as part of the AIPM and STS programs.

Parameter $x_{\max }$ - -A map of gypsy moth counts in pheromone traps (Fig. 6) show numerous spots of increased moth captures that correspond to isolated colonies surrounded by areas with much lower moth counts. Thus, the stratified dispersal approach appears highly appropriate for modeling gypsy moth spread.

Gypsy moth populations reach their carrying capacity when they start defoliating host trees. Thus, the front of the defoliated area was considered as the population front in our model. Sharov et al. (1997) showed that the front of the defoliated area is very unstable in space and time as compared with population boundaries derived from moth captures in pheromone traps and egg mass counts. The boundary of 10 moths per trap (Fig. 6) was the most stable, and therefore, it was most reliable for monitoring population spread. Thus, the distance from a colony to the defoliation front was estimated as the distance to the 10 moths-per-trap boundary plus the average distance from the 10 mothper-trap boundary to the defoliation front which was $80 \mathrm{~km}$ (Sharov et al. 1996).

Gypsy moth colonies detected using pheromone traps cannot be directly interpreted in the model because the majority of them disappear naturally over time, whereas colonies in the model grow continuously. However, we can assume that the number of colonies that eventually become established is proportional to the number of detected colonies. In order to delineate colonies, we selected traps that caught $N$ or more moths and then grouped these traps if they were separated by $<4 \mathrm{~km}$ (4 km was selected because it was larger than the maximum intertrap distance). These groups were counted in each year (1984 and 1988-1995). Colonies may coalesce closer to the population front. Thus, we restricted colony counting to the area beyond the one moth-per-trap line. The coordinates of the center of a colony were estimated as averaged coordinates of all traps that were grouped. Colonies were counted separately in each $50-\mathrm{km}$ distance interval from the pop-

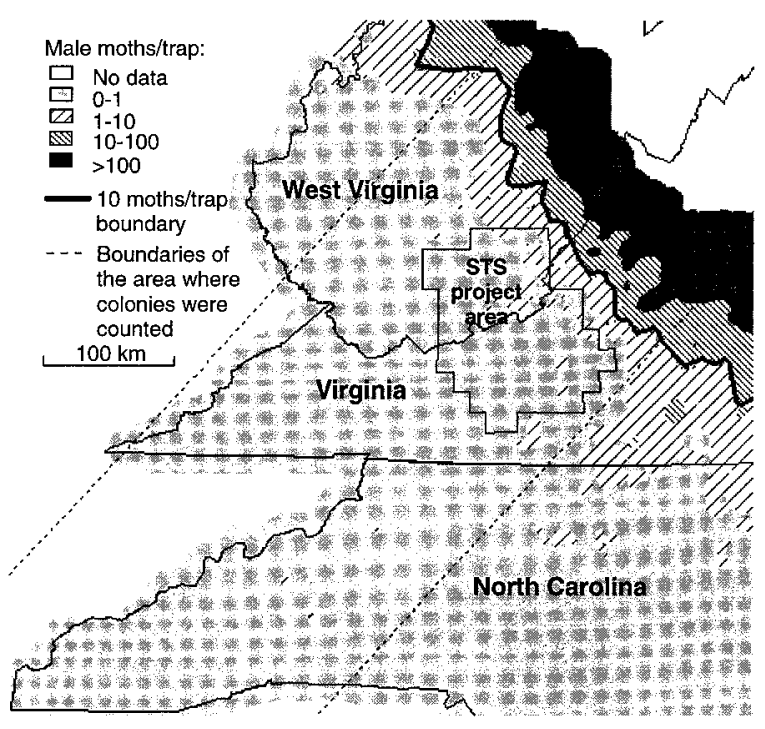

FIG. 6. Gypsy moth counts in pheromone traps in 1995 in West Virginia, Virginia, and North Carolina interpolated using median kriging. 


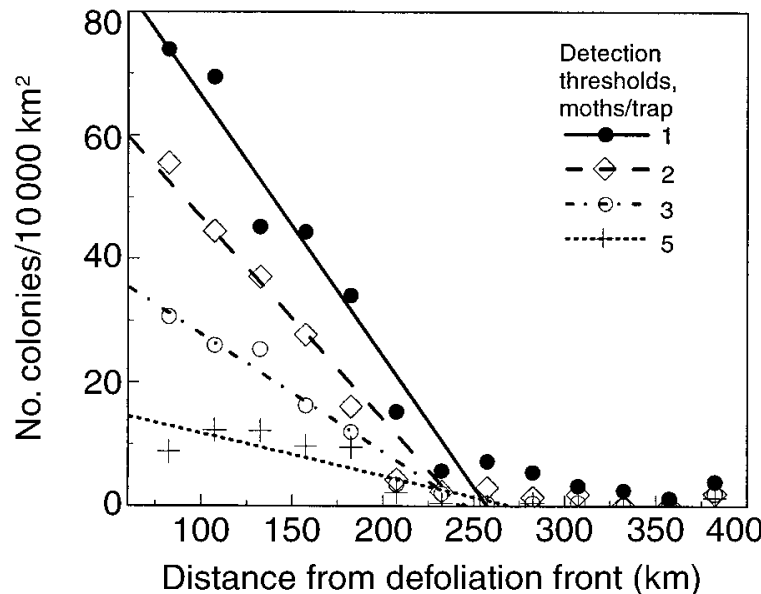

FIG. 7. The number of gypsy moth colonies detected at different distances from the front of the infested zone using $1,2,3$, and 5 moths per trap as detection thresholds.

ulation front. The density of colonies in a distance class $i$ was estimated as $\left(\Sigma_{t} M_{i t}\right) /\left(\Sigma_{t} A_{i t}\right)$, where $M_{i t}$ is the number of colonies detected at distance class $i$ in year $t$, and $A_{i t}$ is the area covered by the pheromone trap grids at distance class $i$ in year $t$. $A_{i t}$ was estimated as the area of a 4-km buffer zone around traps used in the analysis restricted to the distance class $i$.

The number of colonies detected decreased almost linearly with increasing distance from the population front (Fig. 7). Because of the low sensitivity of the model to the shape of function $b(x)$ (Figs. 2 and 3), there was no reason to use more sophisticated nonlinear functions. Lower thresholds, $N$, resulted in a larger number of colonies detected. However, the maximum distance from the defoliation front did not change considerably among male count thresholds and was $\sim 250$ $\mathrm{km}$. Thus, we assumed that $x_{\max }=250 \mathrm{~km}$.

Parameter $c$. - We could not determine parameter $c$ from Fig. 7, because we did not know what proportion of detected colonies would become established. However, we can assume that the rate of colony establishment is the same as the number of eradicated colonies per year per unit area. The number of treatment (pesticides or mating disruption) blocks targeted at isolated colonies in the STS project area in Virginia and West Virginia in 1993-1995 was 5, 12, and 7, respectively, and the project area was 12443,12649 , and 10147 $\mathrm{km}^{2}$, respectively. This makes the average rate of colony eradication (and establishment) in the STS area equal to $0.00068 \cdot \mathrm{yr}^{-1} \cdot \mathrm{km}^{-2}$.

Parameter $c$ equals the rate of colony establishment just beyond the front of the area defoliated. The average distance from the center of the STS project area in Virginia and West Virginia to the boundary of 10 moths per trap was $\sim 70 \mathrm{~km}$ in 1993-1995. Thus, the distance from the STS area to the defoliation front was $\sim 150$ $\mathrm{km}$. Assuming the linear function $b(x)$ (Eq. 4), param- eter $c$ was estimated as $0.00068 \cdot x_{\max } /\left(x_{\max }-150\right)=$ $0.0017 \cdot \mathrm{yr}^{-1} \cdot \mathrm{km}^{-2}$.

Parameter $n_{0}$. - The initial population numbers $n_{0}$ in isolated colonies was not known. We doubt that a colony may start from one egg mass because of the low mating probability (Sharov et al. 1995b). Our expectation of the average initial number of egg masses that may start a new colony is $n_{0}=5$. This parameter is not present in Eq. 15, and thus it does not change the effect of the barrier zone on the rate of spread. However, we used parameter $n_{0}$ for estimating parameter $r$ from Eq. 13.

Parameter K.-Population density at the front of defoliation area, $K$, was taken equal to 200000 egg masses $/ \mathrm{km}^{2}$ because we previously showed that the defoliation boundary (detected at $\sim 30 \%$ level) was mostly located between the boundaries of 10 and 30 egg masses per 0.01-ha plot (Sharov et al. 1996).

Parameter r.-Sharov et al. (1995b) estimated the average intrinsic rate of increase in gypsy moth populations from egg mass samples collected by Campbell and Sloan (1978) in Glenville, New York, USA, in 1958-1963. Only data within the population density interval from 3000 to 300000 eggs/ha were used, because sampling error was too high at densities below 3000 eggs/ha, and populations did not grow exponentially above 300000 eggs/ha. For 25 data points, the average intrinsic rate of population increase was $r=$ 1.65 .

However, all populations in Glenville had considerably higher density than isolated infestations beyond the population front. The extrapolation of $r$ values from high- to low-density populations may be misleading because population dynamics may change with density (Campbell and Sloan 1978, Elkinton and Liebhold 1990). Thus, we decided to get an independent estimate of parameter $r$ using our model of population spread. The value of the parameter $r$ was adjusted until the model gave a rate of population spread that matched rates observed in nature. The rate of uncontrolled population spread (without barrier zones) in areas with a mean minimum January temperature $>-13.8^{\circ} \mathrm{C}$ was $20.78 \mathrm{~km} / \mathrm{yr}$ from 1966 to 1990 (Liebhold et al. 1992). Our study area satisfies this temperature requirement; thus, we can assume that $v_{\max }=20.78 \mathrm{~km} / \mathrm{yr}$. The intrinsic population growth rate, $r=1.706$, was estimated numerically using Eq. 13. This means that gypsy moth populations increased their numbers $\exp (r)=5.51$ times per year.

Both methods resulted in very similar estimates of the parameter $r$. In the model we used the value $r=$ 1.706.

Parameter $x_{0}$-Currently, the barrier zone in the STS project in Virginia and West Virginia starts at a distance of $\sim 20 \mathrm{~km}$ from the 10-moth-per-trap boundary line (Fig. 6). Then, the distance from the start of the barrier zone to the population front is $x_{0}=20+$ $80=100 \mathrm{~km}$. The width of the barrier zone is $\sim 100$ 
km (Fig. 6), whereas the width of the transition zone is $x_{\max }=250 \mathrm{~km}$. Thus, the distant portion $(50-\mathrm{km}$ band) is not covered by the barrier zone. However, isolated colonies are detected and eradicated even beyond the barrier zone by a different agency (USDA Animal and Plant Health Inspection Service). Thus, we can consider that the width of the entire barrier zone is $150 \mathrm{~km}$. Then, $W=150 / 250=0.6$.

Simulation of population spread.-Now we can substitute parameter values into Eq. 10 and estimate the effect of the STS barrier zone in Virginia and West Virginia on the rate of gypsy moth spread. Parameter $Q=r x_{\max } / \nu_{\max }=1.706 \cdot 250 / 20.78=20.52$. Substituting these values into Eq. 10, we predict that the relative reduction of population spread rate $R=0.54$ (54\%).

This value can be compared with the observed reduction in the rate of gypsy moth spread. Attempts to slow the spread of gypsy moth populations started in 1990 as a part of the AIPM project. Most efforts in the AIPM project were directed at suppression of defoliating populations. This activity presumably had a very limited effect on the rate of population spread. The reduction of the rate of population spread was expected mainly from eradication of isolated colonies beyond the expanding population front which started in 1990 . Thus, we compared spread rates before and after 1990 . The annual rate of population spread was estimated as the distance between population boundaries in two consecutive years (Sharov et al. 1995a). The average rate of spread was $26.5 \mathrm{~km} / \mathrm{yr}$ in 1981-1990, and $8.6 \mathrm{~km} /$ yr in 1991-1995 (Sharov and Liebhold 1997). According to Liebhold et al. (1992), the rate of uncontrolled population spread was $20.78 \mathrm{~km} / \mathrm{yr}$ in 1966 1990 which is close to our estimates for 1981-1990. It is not clear which estimate of the uncontrolled spread rate is more accurate. Liebhold et al. (1992) used a less accurate method than our method (the rate of spread was estimated from the boundary of the quarantine zone), but these authors analyzed a longer period. In this paper we use the result of Liebhold et al. (1992) because it gives a more conservative estimate of the reduction in the rate of population spread (59\% reduction). This observed 59\% reduction in the rate of population spread is close to the $54 \%$ predicted by our model.

\section{DISCUSSION}

Generality of the model.-Our model implements a metapopulation approach to the study of population expansion initially suggested by Shigesada et al. (1995). It describes the establishment and growth of isolated colonies rather than dispersal and reproduction of individuals. Instead of the distance traveled by an individual, the model considers the rate of establishment of isolated colonies at varying distances from the population front. Models that describe the movement of individual organisms may seem more attractive, because it is easier to measure dispersing abilities of in- dividuals rather than the rate of establishment of colonies. However, experiments on animal dispersal may yield confusing results, because they do not necessarily represent all possible dispersal mechanisms. For example, the use of experimental measurements of distances traveled under natural conditions by dispersing larvae considerably underestimated the rate of spread of gypsy moth populations (Liebhold et al. 1992) because the major mechanism of spread is transportation of life stages by humans, which is much more difficult to measure directly. The analysis of the rate of colony establishment accounts for all types of dispersal, and thus, it should yield more reliable results.

Another advantage of the metapopulation approach is that it does not use assumptions about reproductive success of dispersing individuals. Models that describe the movement of individual organisms usually assume the same reproductive success in dispersing and nondispersing organisms. However, several factors may reduce the reproductive rate of migrants. First, individuals that disperse beyond the population front may have limited success in finding a mate, because the population density is much lower there. Second, dispersing individuals may land in unfavorable habitats and die. These two factors are very difficult to incorporate in models that describe dispersal of individual organisms, but in a metapopulation model they all are included in the function of the probability of colony establishment, $b(x)$.

The major limitation of our model is that the probability of colony establishment depends on the distance from the population front only. However, the slope of function $b(x)$ may also depend on the slope of the profile of population density across the advancing front which can be measured from the distance between population boundaries estimated at various thresholds. For example, the distance between population boundaries of the gypsy moth (estimated using thresholds of 1,3 , $10,30,100$, and 300 moths per trap) decreased from 1988 to 1994 because of pest management activity in the barrier zone (Sharov et al. 1996). We called this effect "boundary compression." Our model can be used only if the function $b(x)$ is not affected by boundary compression. To satisfy this condition, the maximum distance of long-range dispersal, $x_{\max }$, should be much greater than the distance, $x^{*}$, between the population front and the farthest area that can produce sufficient number of dispersing individuals. In the proximity of the population front, the density of individuals, $N$, increases mostly because of reproduction of local populations rather than colonization by migrants. If the population front advances with a constant velocity, $v_{\max }$, and population numbers grow exponentially with a rate $r$, then the density of individuals declines approximately exponentially with increasing distance from the population front: $N(x) \approx K \exp (-x r)$ $\left.v_{\max }\right)$. At distance $x=3 v_{\max } / r$, the density of individuals becomes $<5 \%$ of the carrying capacity. Thus, we can 
define $x^{*}=3 \cdot v_{\max } / r$. Then, our model can be used on condition that $x_{\max } \gg 3 v_{\max } / r$. Gypsy moth spread satisfies this condition because $x_{\max }=250 \mathrm{~km}$, and $3 v_{\max } /$ $r=36.6 \mathrm{~km}$.

Another constraint is that the model may underestimate the rate of population spread if the barrier zone is placed too close to the population front. In this case, the population will spread continuously rather than by establishment of isolated colonies. Also, the model assumes a $100 \%$ success in eradication of isolated colonies in the barrier zone, which is not always possible. We think that this model is most appropriate for simulating a moderate reduction of the population spread rate (e.g., by $<70 \%$ ). In this case, the barrier zone would be located relatively far from the population front; thus, the problems described above will not apply.

We are not aware of data on the probability of colony establishment beyond the population front in other species besides the gypsy moth. Thus, we could not apply our model to estimate the rate of spread in other species. Detection of isolated colonies requires extensive sampling programs that use highly sensitive methods such as pheromone traps as used for the gypsy moth. We expect that this model will be useful mostly for pest species that are important enough to justify an intensive monitoring program.

Model sensitivity.-The rate of spread of uncontrolled populations predicted by the model was proportional to the maximum distance at which colonies can become established. The rate of population increase, $r$, had a stronger effect on the rate of spread in our model than in the model of Skellam (1951), which predicts that the rate of spread is proportional to the square root of the rate of population increase. Our model predicts an almost a linear relationship between parameter $r$ and the rate of population spread (Fig. 2). This effect results from a relatively small proportion of recent migrants in the area located just beyond the population front. The increase in population density in this area results mostly from the growth of local populations rather than from immigration. As a result, the effect of parameter $r$ is stronger than in the model of Skellam (1951) in which migrating organisms have a greater contribution to the growth of population numbers.

The model suggests that the reduction of colonization rate, $c$, has a very limited effect on the rate of population spread. We showed that a 100 -fold decrease in colonization rate may result in only a twofold decrease in spread rates. An important implication of this result is that quarantine regulations may not always be an efficient mean to reduce the rate of population spread. When the colonization rate is high, and the term $c n_{0} / K$ is large enough, then quarantine regulations are justified. However, when the colonization rate is low, then its further reduction may have a very limited effect on the rate of spread. In this case, it may be better to invest resources into barrier zones rather than into intensification of the quarantine.

The shape of function $b(x)$ (linear-, quadratic-, and 1/2-normal distribution function) had a limited effect on the rate of population spread. Thus, using the most simple linear function is satisfactory for the analysis.

Effect of barrier zones on the rate of spread.-Previous models of population spread were aimed only at predicting the natural rate of spread. Our model can predict how to modify the rate of spread using barrier zones, and thus, it can be used for population management. The model indicates that the barrier zone established by the STS project can reduce the rate of spread of gypsy moth populations in the Appalachian Mountains by $54 \%$. This prediction was close to the $59 \%$ reduction in the rate of gypsy moth spread observed since 1990.

However, we should be cautious in interpreting this coincidence. First, the barrier zone strategy was implemented consistently only in the STS project that started in 1992. In the previous AIPM project, eradication of small isolated colonies was conducted simultaneously with intensive suppression of outbreak populations, which may have also contributed to the reduction of gypsy moth spread rate in the area by reducing $c$. Second, population spread rate has historically fluctuated considerably (Sharov et al. 1996). From 1991 to 1995 , it ranged from 4.6 to $14.3 \mathrm{~km} / \mathrm{yr}$ (Sharov and Liebhold 1997). Thus, the accuracy of the observed reduction in the rate of gypsy moth spread is not high.

This model is too abstract for developing a detailed plan of slowing the spread of any species because it does not consider the density of samples used for monitoring, the cost of sampling and treatment activities, and many other details. In this paper we present the model in its general form, whereas a detailed model designed for management of gypsy moth spread is presented in another paper (Sharov et al. 1998).

\section{ACKNOWLEDGMENTS}

We thank E. Anderson Roberts (Virginia Polytechnic Institute and State University, Blacksburg, Virginia) for providing gypsy moth data, and Alan Hastings (University of California, Davis, California) for reviewing the manuscript. This research has been funded by USDA Forest Service, Forest Health, contract No. 08-94-53-01 and by Grant No. 9537313-1892 from the USDA, Cooperative State Research, Education, and Extension Service.

\section{Literature Cited}

Campbell, R. W., and R. J. Sloan. 1978. Numerical bimodality among North American gypsy moth populations. Environmental Entomology 7:641-646.

Collins, C. W. 1915. Dispersion of gypsy-moth larvae by the wind. U.S. Department of Agriculture Bulletin 273:1-62.

Dahlsten, D. L., and R. Garcia. 1989. Eradication of exotic pests. Analysis with case histories. Yale University Press, New Haven, Connecticut, USA.

Elkinton, J. S., and A. M. Liebhold. 1990. Population dynamics of gypsy moth in North America. Annual Review of Entomology 35:571-596. 
Hanski, I., and M. Gilpin. 1991. Metapopulation dynamics: brief history and conceptual domain. Biological Journal of the Linnean Society 42:3-16.

Hastings, A. 1991. McKendrich Von Foerster models for patch dynamics. Lecture Notes in Biomathematics. 92:189_ 199.

Hengeveld, R. 1989. Dynamics of biological invasions. Chapman and Hall, London, UK.

Leonard, D. S., and A. A. Sharov. 1995. Slow the Spread project update: developing a process for evaluation. Pages 82-85 in Proceedings. U.S. Department of Agriculture interagency gypsy moth research forum 1995. U.S. Forest Service General Technical Report NE-213.

Leuschner, W. A., J. A. Young, S. A. Walden, and F. W. Ravlin. 1996. Potential benefits of slowing the gypsy moth's spread. Southern Journal of Applied Forestry 20: 65-73.

Liebhold, A. M., V. Mastro, and P. W. Schaefer. 1989. Learning from the legacy of Leopold Trouvelot. Bulletin of the Entomological Society of America 35:20-21.

Liebhold, A. M., J. A. Halverson, and G. A. Elmes. 1992. Gypsy moth invasion in North America: a quantitative analysis. Journal of Biogeography 19:513-520.

Liebhold, A. M., W. L. Macdonald, D. Bergdahl, and V. C. Mastro. 1995. Invasion by exotic forest pests: a threat to forest ecosystems. Forest Science Monographs 30. Bethesda, Maryland, USA.

Marsula, R., and C. Wissel. 1994. Insect pest control by a spatial barrier. Ecological Modelling 75/76:203-211.

Mason, C. J., and M. L. McManus. 1981. Larval dispersal of the gypsy moth. Pages 161-202 in C. C. Doane and M. L. McManus, editors. The gypsy moth: research toward integrated pest management. U.S. Department of Agriculture Technical Bulletin $\mathbf{1 5 8 4}$.

McFadden, M. W., and M. E. McManus. 1991. An insect out of control? The potential for spread and establishment of the gypsy moth in new forest areas in the United States. Pages 172-186 in Y. N. Baranchikov, W. J. Mattson, F. P. Hain, and T. L. Payne, editors. Forest insect guilds: patterns of interaction with host trees. U.S. Department of Agriculture Forest Service General Technical Report NE-153.

McManus, M. L., and T. McIntyre. 1981. Introduction. Pages 1-7 in C. C. Doane and M. L. McManus, editors. The gypsy moth: research toward integrated pest management. U.S Department of Agriculture Technical Bulletin 1584.

Mollison, D. 1977. Spatial contact models for ecological and epidemic spread. Journal of the Royal Statistical Society B 39:283-326.

Reardon, R. C. 1991. Appalachian gypsy-moth integrated pest-management project. Forest Ecology and Management 39: $107-112$.
Scruggs, C. G. 1975. The peaceful atom and the deadly fly. Jenkins Publishing, Austin, Texas, USA.

Sharov, A. A., and A. M. Liebhold. 1997. Quantitative analysis of gypsy moth spread in the Central Appalachians. Pages 99-110 in J. Braumgartner, P. Brandmayer, and B. F. J. Manly, editors. Population and Community Ecology for Insect Management and Conservation. Balkema, Rotterdam, The Netherlands.

Sharov A. A., A. M. Liebhold, E. A. Roberts, and F. W. Ravlin. 1995a. Gypsy moth (Lepidoptera: Lymantriidae) spread in the Central Appalachians: Three methods for species boundary estimation. Environmental Entomology 24: $1529-1538$

Sharov, A. A., A. M. Liebhold, and F. W. Ravlin. $1995 b$. Prediction of gypsy moth (Lepidoptera: Lymantriidae) mating success from pheromone trap counts. Environmental Entomology 24:1239-1244.

Sharov A. A., A. M. Liebhold, and E. A. Roberts. 1996. Spread of gypsy moth (Lepidoptera: Lymantriidae) in the Central Appalachians: comparison of population boundaries obtained from male moth capture, egg mass counts, and defoliation records. Environmental Entomology 25: 783-792.

Sharov, A. A., A. M. Liebhold, and E. A. Roberts. 1997. Methods for monitoring the spread of gypsy moth (Lepidoptera: Lymantriidae) populations in the Appalachian Mountains. Journal of Economic Entomology 90:12591266.

Sharov A. A., A. M. Liebhold, and E. A. Roberts. 1998. Optimizing the use of barrier zones to slow the spread of gypsy moth (Lepidoptera: Lymantriidae) populations in North America. Journal of Economic Entomology 91:165174.

Shigesada, N., K. Kawasaki, and Y. Takeda. 1995. Modeling stratified diffusion in biological invasions. American Naturalist 146: 229-251.

Skellam, J. G. 1951. Random dispersal in theoretical populations. Biometrika 38:196-218.

Taylor, R. A. J., and D. Reling. 1986. Density/height profile and long-range dispersal of first-instar gypsy moth (Lepidoptera: Lymantriidae). Environmental Entomology 15: 431-435.

Van den Bosch, F., J. A. J. Metz, and O. Diekman. 1990. The velocity of spatial population expansion. Journal of Mathematical Biology 28:529-565.

Van den Bosch, F., R. Hengeveld, and J. A. J. Metz. 1992. Analyzing the velocity of animal range expansion. Journal of Biogeography 19:135-150.

Wilder, J. W., I. Christie, and J. J. Colbert. 1995. Modelling of two-dimensional spatial effects on the spread of forest pests and their management. Ecological Modelling 82:287298. 